\title{
Cell therapy for the treatment of sepsis and acute respiratory distress syndrome
}

\author{
Raquel Guillamat-Prats $^{1,2}$, Marta Camprubí-Rimblas ${ }^{1,2,3}$, Josep Bringué ${ }^{1,2,3}$, Neus Tantinyà ${ }^{1,2}$, Antonio \\ Artigas $1,2,3,4$
}

${ }^{1}$ Centro de Investigaciones Biomédicas en Red de Enfermedades Respiratorias (CIBERES), Madrid, Spain; ${ }^{2}$ Institut d'Investigació i Innovació Parc Taulí (I3PT), Sabadell, Catalonia, Spain; ${ }^{3}$ Universitat Autonoma de Barcelona, Bellaterra, Catalonia, Spain; ${ }^{4}$ Critical Care Center, Corporació Sanitària i Universitària Parc Taulí, Sabadell, Catalonia, Spain

Contributions: (I) Conception and design: All authors; (II) Administrative support: All authors; (III) Provision of study materials or patients: All authors; (IV) Collection and assembly of data: All authors; (V) Data analysis and interpretation: All authors; (VI) Manuscript writing: All authors; (VII) Final approval of manuscript: All authors.

Correspondence to: Antonio Artigas. Fundació Parc Taulí, C/Parc Taulí, 1, 08208, Sabadell, Spain. Email: aartigas@tauli.cat.

\begin{abstract}
Sepsis and acute respiratory distress syndrome (ARDS) are life threating diseases with high mortality and morbidity in all the critical care units around the world. After decades of research, and numerous pre-clinical and clinical trials, sepsis and ARDS remain without a specific and effective pharmacotherapy and essentially the management remains supportive. In the last years cell therapies gained potential as a therapeutic treatment for ARDS and sepsis. Based on numerous pre-clinical studies, there is a growing evidence of the potential benefits of cell based therapies for the treatment of sepsis and ARDS. Several cell types are used in the last years for the treatment of both syndromes showing high efficiency. Embryonic stem cells (ESC), multipotent stem (or stromal) cells (MSC) and epithelial progenitors cells (EpPC) have been used for both diseases. Nowadays, the major part of the pre-clinical studies are using MSC, however other relevant groups are also using induced pluripotent stem cells (iPSC) for the treatment of both syndromes and alveolar type II cells for ARDS treatment. Numerous questions need further study including: determining the best source for the progenitor cells isolation, their large scale production and cryopreservation. Also, the heterogeneity of patients with sepsis and ARDS is massive, and establish a target population or the stratification of the patients will help us to determine better the therapeutic effect of these cell therapies. In this review we are going to describe briefly the different cell types, their potential sources and characteristics and mechanism of action. Here, also we elucidate the results of several pre-clicinical and clinical studies in ARDS and in sepsis and the future directions of these studies.
\end{abstract}

Keywords: Sepsis; acute respiratory distress syndrome (ARDS); acute lung injury; cell therapy; critical care

Submitted Jul 10, 2017. Accepted for publication Aug 14, 2017.

doi: 10.21037/atm.2017.08.28

View this article at: http://dx.doi.org/10.21037/atm.2017.08.28

\section{Introduction}

Sepsis and acute respiratory distress syndrome (ARDS) are life threating diseases with high mortality and morbidity in all the critical care units around the world. Severe sepsis is a complex syndrome produced by the response to a systemic infection $(1,2)$. The infection produces a general inflammatory response, such as tachycardia, elevated white-cell count and systemic release of pro-inflammatory cytokines, and this can lead to an acute organ dysfunction $(3,4)$. The lung is one of the most affected organs during sepsis, and for that reason, one of the main indirect causes of ARDS is sepsis (4). ARDS can also be produced by a direct injury as a pulmonary infection or a trauma $(5,6)$. ARDS is a multifactorial syndrome characterized by increased lung permeability, hypoxemia, the absence of cardiogenic 
Table 1 Summary of the cell sources and their benefits

\begin{tabular}{|c|c|c|c|c|c|}
\hline Cell type & Harvest method & Advantages & Disadvantages & Benefits in ARDS & Benefits in sepsis \\
\hline iPSC & Skin biopsy & $\begin{array}{l}\text { Easy isolation. No } \\
\text { rejection }\end{array}$ & High tumorigenic potential & $\begin{array}{l}\text { Easy to differentiate to AEC2. } \\
\text { No tested in vivo }\end{array}$ & - \\
\hline EnPC & Blood & Non tumorigenic & $\begin{array}{l}\text { Difficult isolation and small } \\
\text { amount }\end{array}$ & $\begin{array}{l}\text { Maintain the integrity of the } \\
\text { lung and improve the lung } \\
\text { function }\end{array}$ & $\begin{array}{l}\text { Reduce the sepsis damage } \\
\text { re-establishing micro and } \\
\text { macrocirculation }\end{array}$ \\
\hline
\end{tabular}

ARDS, acute respiratory distress syndrome; ESC, embryonic stem cells; iPSC, induced pluripotent stem cells; MSC, multipotent stem (or stromal) cells; EnPC, endothelial progenitor cells ; EpPC, epithelial progenitors cells.

pulmonary edema, the disruption of the alveolar-capillary barrier and widespread inflammation $(7,8)$.

After decades of research, and numerous pre-clinical and clinical trials, sepsis and ARDS remain without a specific and effective pharmacotherapy and essentially the management remains supportive $(4,8)$.

On the last two decades, several groups started working in cell therapies and gaining potential as a therapeutic treatment for ARDS and sepsis. Based on numerous preclinical studies, there is a growing evidence of the potential benefits of cell based therapies for the treatment of sepsis and ARDS. Several cell types are used in the last years for the treatment of both syndromes showing high efficiency. This review summarizes the different progenitor cells that can be used as a therapy, the mechanisms of action and the results in pre-clinical and clinical studies in ARDS and in sepsis and future directions.

\section{Different cell-based therapies}

Embryonic stem cells (ESC), multipotent stem (or stromal) cells (MSC) and epithelial progenitors cells (EpPC) have been used for the treatment of sepsis and ARDS. Nowadays, the major part of the pre-clinical studies are using MSC, however other relevant groups are also using induced pluripotent stem cells (iPSC) for the treatment of both syndromes and alveolar type II cells for ARDS treatment.
We are going to review briefly the different cell types, their potential sources and characteristics; all of these is summarized in the Table 1 and represented in the Figure 1.

\section{ESC}

ESCs are pluripotent cells derived from the inner blastocyst cell mass and constitute a potentially unlimited source of cells that could be differentiated into any progenitor cell and that could be used in the clinical $(9,10)$. ESC could give rise to all cell types following therapeutic transplantation, and this will allow the complete regeneration of the tissue. Because of their plasticity and theoretically unlimited capacity for self-renewal, ESCs have been suggested for regenerative medicine and tissue replacement after injury or disease (11). However, their embryologic origin is linked to significant ethical issues regarding the use of these cells. Many crucial questions remain to be addressed before the full potential of ESC can be applied.

\section{iPSC}

iPSCs are a new type of pluripotent cells that can be obtained by reprogramming animal and human somatic (differentiated) cells. iPSC can be obtained from dermal fibroblasts that have suffered dedifferentiation following reprogramming and finally are able to express four 


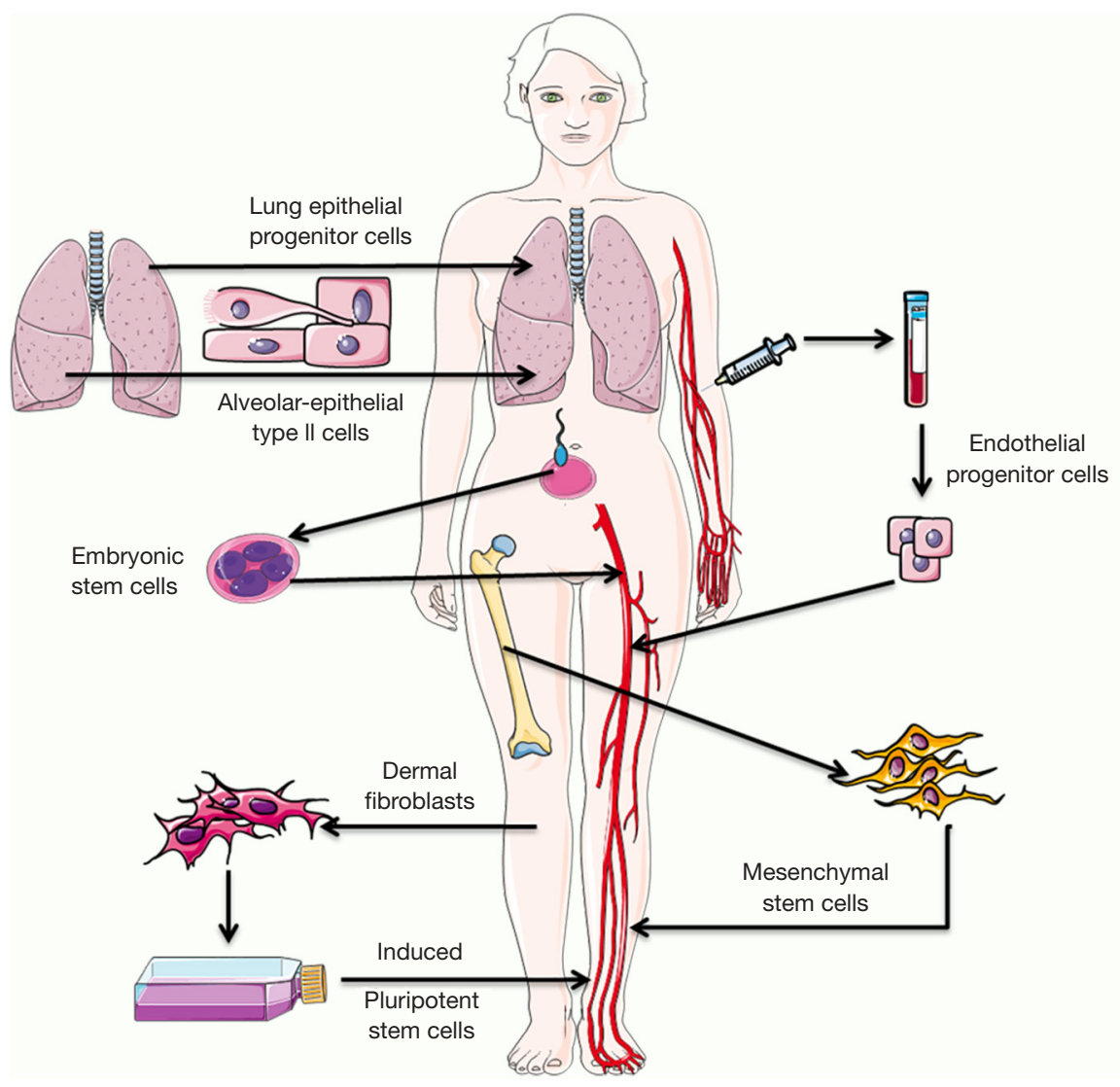

Figure 1 Potential sources of progenitor cells.

transcription factors, Ocet $3 / 4$, Sox 2 , Klf- 4 and c-Myc $(12,13)$. These cells can be obtained in autologous way eliminating the problem of immune rejection associated with other cells. They have the potential of growing indefinitely and differentiating into all cell types of the human body (14). The potential applications of iPSCs in sepsis and ARDS are numerous. However, the dedifferentiation and reprogramming have low efficiency, and their genomic modification could create associated problems that until now are unknown or have not been studied and have a high tumorigenicity risk (15).

\section{Endothelial progenitor cells (EnPC)}

The endothelial damage is one of the main hallmarks of ARDS and EnPCs have the ability to regenerate endothelial tubes and could have an unlimited role in repairing the damaged endothelium. EnPcs have been defined as circulating cells that express hematopoietic surface cell markers such as CD34 and have the ability to adhere to the endothelium at sites of hypoxia and ischemia secrete proangiogenic factors and generate a new vessel (16). Also, the role, isolation and identification of these cells are not completly elucidated. These cells might be a regenerative tool for the treatment of vascular diseases and to restrict angiogenesis in tumors. Nevertheless, the role of EnPC in vascular biology is still in debate and conflicting results have been published $(17,18)$. More investigations are required; a few pre-clinical and clinical trials using EnPCs have been published and it is necessary to explore more areas and therapeutic potential of EnPCs.

\section{EpPC}

EpPCs are specified during development in each tissue and are highly regulated by epithelial-mesenchymal interactions. There are several functional variances in EpPCs amongst the different tissues, but their maintenance, activation and differentiation is regulated by the same pathways between in all the tissues (19). The deregulation of some of these 


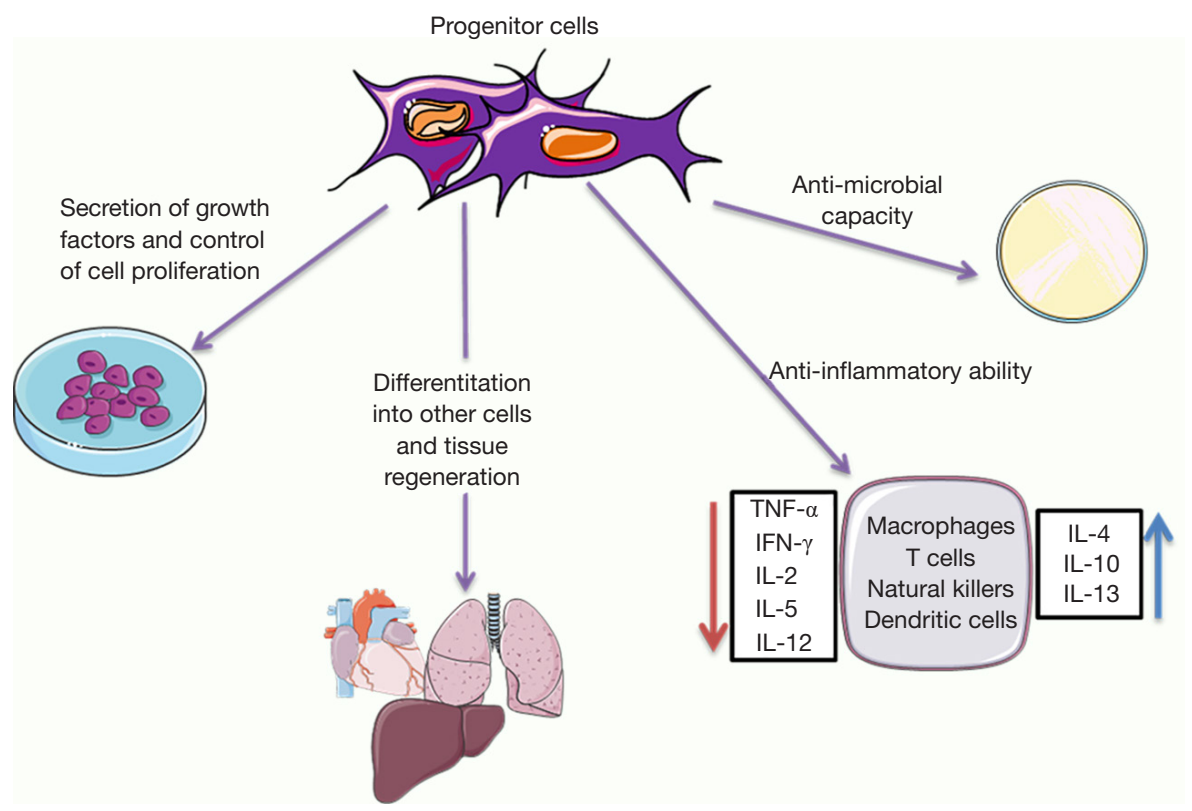

Figure 2 Schematic diagram demonstrating the potential mechanisms of progenitor cells. Some of them can modulate the immune response, have antibacterial properties and can differentiate to several tissues regenerating them.

pathways can origin some pathological disorders such as cancer. Some epithelial progenitors of the lung alveolar compartment have also been identified $(20,21)$. However, their isolation is really difficult and the number of cells that could be obtained is really low.

Another strategy is the use of alveolar-epithelial type II cells (AEC2); these cells are more differentiated than EPCs, but still can proliferate and differentiate into alveolarepithelial type I cell, that are the complete differentiated cells in the lung epithelia $(22,23)$. AEC2 cells could be isolated from the lungs of organ donors and have less ethical problems and tumorogenicity potential. However their isolation is not easy and their use as therapy should be always associated to the rejection problem.

\section{Mesenchymal stem (or stromal) cells}

MSCs are the best described cells and the most used as a cell therapy. The International Society of Cellular Therapy defined that MSCs should follow three criteria: (I) MSCs must be adherent to plastic; (II) MSCs must express some cell surface markers, such as CD105, CD90 and CD73, but must not express other markers, including CD45, CD34, CD14 or CD11b; and (III) MSCs must have the capacity to differentiate into mesenchymal lineages (osteoblasts, adipocytes and chrondoblasts) in in vitro conditions (24).

MSC are multipotent cells that have been isolated from several tissues such as umbilical cord blood, placenta, adipose tissue, lung and bone marrow $(25,26)$. MSCs have a high degree of plasticity and can be differentiate into a variety of cell lineages, but they do not possess the complete plasticity of ESCs. However, MSCs have some advantages because of their easy isolation and enormous propagation in culture and also because their use does not involve the ethical problems associated to the use of ESCs $(27,28)$. Moreover, they can be obtained autologous diminishing the immune rejection problem. Several experimental studies have indicated that MSCs may have potential therapeutic application in sepsis and ARDS. It has also been reported that MSC release several micro-vesicles that might have therapeutic potential (29).

\section{Cell therapies in pre-clinical research}

In this second part of this review we are going to discuss the safety and efficacy of all these progenitor cells in the treatment of sepsis or ARDS. Here, we are going to point out the most relevant pre-clinical studies using cell therapy in these two syndromes and the most significant results (Figure 2). 


\section{Use of cell therapies for the treatment of ARDS}

During ARDS, endogenous stem cells participate in the remodeling of the injured lung. The EPCs resident in the lung and the circulating epithelial, endothelial and mesenchymal cells migrate to the lung and try to contribute to the re-epithelialization. However, sometimes endogenous cells are not enough to re-establish the correct function of the lung, and then, exogenous progenitor cells can be used as a therapy.

On the one hand, ESCs or iPSCs offer the possibility to differentiate into any cell of the lung and replace any damaged cell. ESCs were differentiated in vitro to AEC2 by Rippon et al. $(30,31)$ and the differentiated cells were able to express all the normal markers of the AEC2, however they were never tested in vivo. Similarly, iPSC were differentiated into AEC2 for several groups, but their effect was also not tested in vivo (32-34). The pluripotency of ESC and iPSC and their ability to proliferate indefinitely without differentiating increases the risk of a neoplasia and for that reason their use in in vivo models is really limited; researchers are concerned about the restrictions of their use.

On the other hand, MSCs have more immunomodulatory potential; they are able to reprogram the immune system and reduce inflammation. MSCs have been widely used for the treatment of direct and indirect ARDS in several in vivo models. Moreover, MSCs were described effective to reduce ARDS induced by a ventilator (VILI) (35-37), sepsis $(38,39)$ or pneumonia $(40,41)$. It has been described by several groups that MSCs are not engrafted and differentiates to lung alveolar-epithelial cells; simply, they are doing their effect following paracrine mechanisms. MSC seems to release several mediators such as miRNAs, mitochondria, proteins and acids nucleic directly or via microvesicles and exosomes that are able to modulate other cells such as macrophages, dendritic cells, neutrophils, natural killers, alveolar-epithelial cells and T and B lymphocytes.

We can find in literature more than 30 papers published in the last decade indicating that MSCs reduce mortality and improve several clinical course indicators (39,42-44). Also, it has been extensively described that MSCs decrease the expression of several pro-inflammatory cytokines such as TNF- $\alpha$, IL- $1 \beta$, IL- 6 , and IFN- $\gamma$ and increase antiinflammatory cytokines such as IL-4 and IL-10 $(39,43,44)$. At the end, the resolution of the ARDS is improved by the release of several paracrine factors produced by MSC that restore lung function.
EnPCs were also tested in animal models with ARDS and these progenitor cells were also able to maintain the integrity of the lung epithelium and improve the lung function $(45,46)$. EnPCs reduced inflammation via the reduction of IL- $1 \beta$ and increased anti-inflammatory IL-10 expression $(47,48)$.

To finish this section, we want to highlight the preliminary results of the direct treatment of ARDS with AEC2. These cells are more differentiated than other cells and their progenitor function is limited, however, their possible tumorigenicity and transdifferentiation to cells that we do not want might be also less than ESC, iPSC and MSCs. AEC2 therapy also increase survival, lung function and reduces pulmonary inflammation (49).

The major part of all these studies cited here, administered the progenitor cells via intratracheal in the lung and usually in the first hours after the induction of ARDS. However, the variety of species, doses, and parameters evaluated makes difficult to obtain a common conclusion; however all of the studies showed an improvement in the lung function diminishing ARDS.

\section{Use of cell therapies for the treatment of sepsis}

The therapeutic potential of the different progenitor cells was widely tested in a cecal and ligation puncture (CLP) model in rat and mice that origins a polymicrobial sepsis. These progenitor cells were always administered systemically by an intravenously administration.

As we explained previously in ARDS section, ESCs or iPSCs offer the possibility to migrate and differentiate into any tissue, giving them a huge potential and also a high risk. As far as we observed, there is only one preclinical study published in sepsis in which ESCs have been used (50), any there is none using iPSC. Toya et al. injected human ESC one hour after cecal ligation and puncture and they described that transplantation reduce mortality in a $40 \%$ and decreases lung inflammation and edema as well as production of tumor necrosis factor- $\alpha(\mathrm{TNF}-\alpha)$ and interferon- $\gamma$ in lungs (IFN- $\gamma$ ) (50).

MSCs are in sepsis, as well as in ARDS, the most used cells. Their easy isolation and expansion make them perfect for a therapy. MSCs treatment of pre-clinical sepsis significantly reduced mortality $(39,44,51,52)$. Also it was reported that MSCs improved bacteria clearance; they do not have phagocytic activity $(44,51,53)$, nevertheless they are able to induce phagocytosis of macrophages $(41,44)$, neutrophils (54) and peripheral monocytes (53). 
MSCs administration in septic animals also resulted in decreased levels of IL-1 $\alpha$, IL- 6 , and IFN- $\gamma$ in plasma compared to septic animals. No changes usually are observed in IL-4 or IL-10 (39,55). In several papers it has been reported the anti-apoptotic effect of MSC for example in host neutrophils or monocytes. This effect seems that is mediated by the production of the anti-apoptotic cytokine interleukin and the factor growth factor-7 (FGF7) $(53,56)$. The combination of reducing inflammation and apoptosis leads to a decrease in the multiple organ failure, also described as an effect of the MSC therapy for several groups. Mostly, it is well described that animals treated with MSC developed less ARDS or the ARDS is less destructive (57), moreover other studies described also less acute kidney injury or liver injury after the MSC treatment (58-60). In summary, sepsis initiate endothelial damage caused by an increased apoptosis and inflammation and altogether leads to an organ failure; the use of MSC is able to stop this cascade at several points and is a promising alternative for the sepsis treatment.

The combination of MSC with other treatments such as antibiotics administration $(44,61)$ or the application of novel techniques such as extracorporeal membrane oxygenation (ECMO) may give us more fascinating results in a close future (62).

EnPCs were also tested for the sepsis treatment with positive results. Sepsis alters endothelial function in the macro and microcirculation and causes damage in the endothelial barrier, so the treatment with EnPCs seems interesting to reduce the sepsis damage. In three published studies with EnPC, their administration reduced mortality and down-regulated TLR4, TNF- $\alpha$, IL- $1 \beta$, IL- 6 and IL-10 levels (63-65). EnPC transplantation was able to diminish significantly lung, liver, and kidney tissue damage $(63,64)$.

Overall, cell therapies are able to improve survival, reducing inflammation and apoptosis, improving the clearance of bacteria and producing less organ failure. To go in deep into the several mechanisms is necessary to determine which population is better in each situation and could cause fewer side effects.

\section{Clinical trials with cell therapy in sepsis and ARDS}

At the moment, there are several ongoing clinical trials registered, mainly using MSCs. A few numbers of reports describing results have been published. One of the earliest studies was conducted by Zheng et al and they studied the safety of MSCs in ARDS patients. In this phase I, 12 patients were randomized and received an intravenous administration of either $100 \mathrm{~mL}$ saline or a single dose of allogeneic MSCs obtained from adipose tissue (66). The authors reported that the cell therapy was safe, but they did not find significant differences in total length of hospital stay, ICU-free days, and ventilator-free days or in serum ARDS biomarkers. Another trial conducted by Galstyan et al. with 27 patients (13 received conventional treatment while 14 received conventional treatment plus a single dose of $1 \times 10^{6} \mathrm{MSC}$ ) was performed to assess the effect of MSC in sepsis (septic shock) (67). The group treated with MSCs had a significant increase in 28-day survival rates $(57 \%$ vs. $15 \%)$ that was associated with a decrease in SOFA-score. However, there was no difference in post-28 day survival rates. Wilson et al. presented results of a phase I, multicenter, open label, dose-escalation pilot study, (START) in patients with moderate to severe ARDS (68). The study demonstrated that allogeneic MSC isolated from bone marrow were well tolerated, however they did not find any change in the pro-inflammatory markers. The results from all of these clinical trials have provided promising results that MSCs can offer a therapeutic benefit.

Nowadays, there are in progress several phase 2a clinical trials to establish safety in ARDS and similarly in sepsis. In the Table 2 we summarized the results of phase 1 clinical trials and we report the ongoing phase 2 a clinical trials.

\section{Conclusions}

Still, numerous questions need further study including: determining the best source for the MSCs isolation, their large scale production and cryopreservation. Moreover, the therapeutic potential of MSCs and its conditioned media need to be studied for checking their efficacy in short term and long term follow up studies. A lot of work for the better characterization and potential of the several progenitor cells need to be performed.

The heterogeneity of patients with sepsis and ARDS is massive, and establish a target population or the stratification of the patients will help us to determine better the therapeutic effect of these cell therapies. Furthermore, define better biomarkers and evaluate them in all the future clinical trials at the same time points will give us an extensive view of the benefits of cell therapies. Probably, the patients in a hyperactive inflammatory phase may have more benefits of these therapies and will show more improvement. 


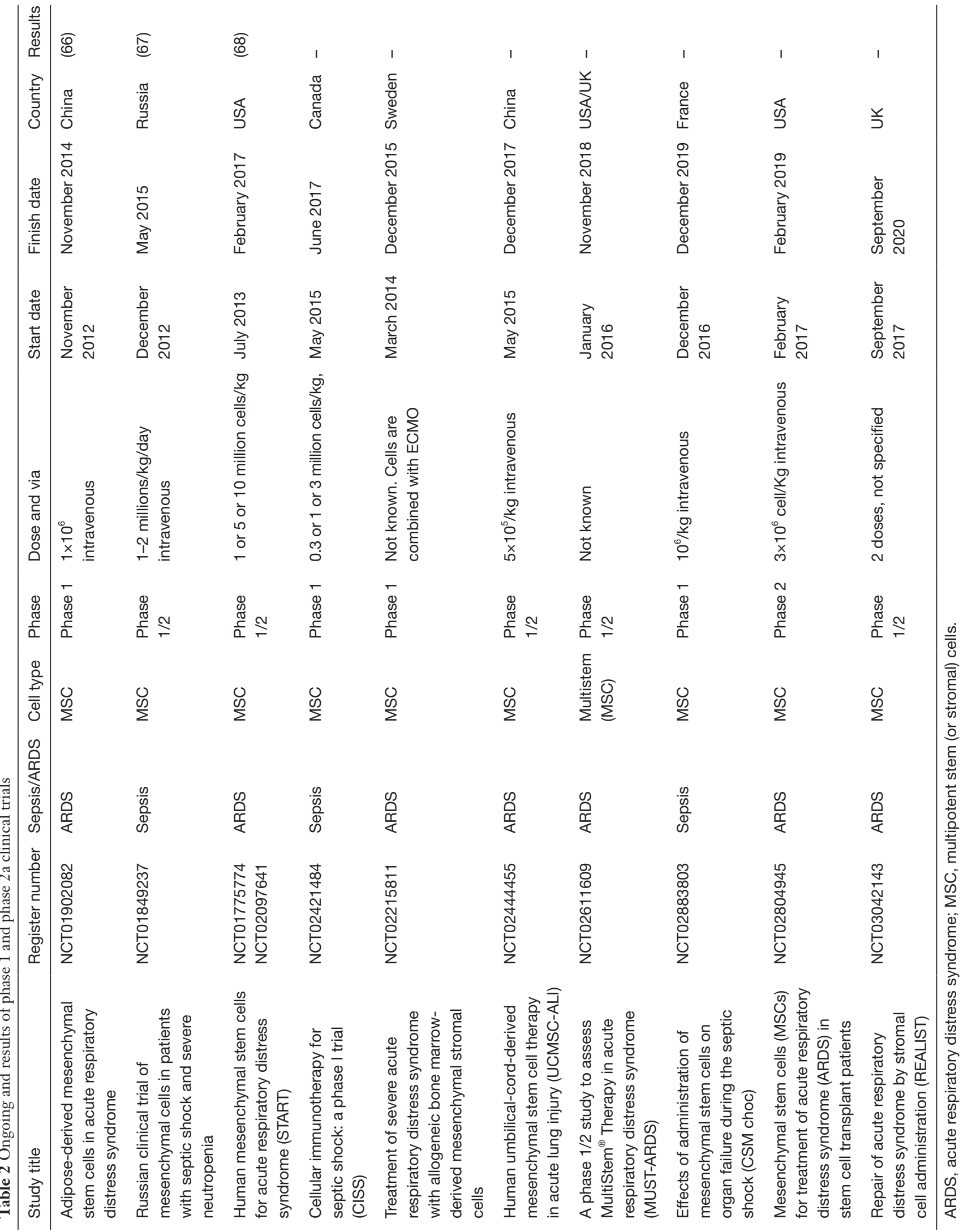




\section{Acknowledgements}

Funding: This work was supported by Ministerio de Economía y Competitividad-Instituto de Salud Carlos III (PI12/02548 for A Artigas) Cofinanciado por el Fondo Europeo de Desarrollo Regional (FEDER), Beca becari SOCAP (for M Camprubí-Rimblas) and CIBER de Enfermedades Respiratorias (CIBERES).

\section{Footnote}

Conflicts of Interest: The authors have no conflicts of interest to declare.

\section{References}

1. Levy MM, Fink MP, Marshall JC, et al. 2001 SCCM/ ESICM/ACCP/ATS/SIS International Sepsis Definitions Conference. Intensive Care Med 2003;29:530-8.

2. Freund Y, Lemachatti N, Krastinova E, et al. Prognostic accuracy of sepsis-3 criteria for in-hospital mortality among patients with suspected infection presenting to the emergency department. JAMA 2017;317:301-8.

3. Angus DC, van der Poll T. Severe sepsis and septic shock. N Engl J Med 2013;369:2063.

4. Martin-Loeches I, Levy MM, Artigas A. Management of severe sepsis: advances, challenges, and current status. Drug Des Devel Ther 2015;9:2079-88.

5. Luo L, Shaver CM, Zhao Z, et al. Clinical predictors of hospital mortality differ between direct and indirect ARDS. Chest 2017;151:755-63.

6. Matthay MA, Ware LB, Zimmerman GA. The acute respiratory distress syndrome. J Clin Invest 2012;122:2731-40.

7. Rubenfeld GD, Caldwell E, Peabody E, et al. Incidence and outcomes of acute lung injury. $\mathrm{N}$ Engl J Med 2005;353:1685-93.

8. Ware LB, Matthay MA. The acute respiratory distress syndrome. N Engl J Med 2000;342:1334-49.

9. Martin GR, Evans MJ. Differentiation of clonal lines of teratocarcinoma cells: formation of embryoid bodies in vitro. Proc Natl Acad Sci U S A 1975;72:1441-5.

10. Thomson JA, Itskovitz-Eldor J, Shapiro SS, et al. Embryonic stem cell lines derived from human blastocysts. Science 1998;282:1145-7.

11. Yu J, Thomson JA. Pluripotent stem cell lines. Genes Dev 2008;22:1987-97.

12. Takahashi K, Yamanaka S. Induction of pluripotent stem cells from mouse embryonic and adult fibroblast cultures by defined factors. Cell 2006;126:663-76.

13. Okita K, Ichisaka T, Yamanaka S. Generation of germlinecompetent induced pluripotent stem cells. Nature 2007;448:313-7.

14. Scudellari M. How iPS cells changed the world. Nature 2016;534:310-2.

15. Heffernan C, Sumer H, Verma PJ. Generation of clinically relevant "induced pluripotent stem" (iPS) cells. J Stem Cells 2011;6:109-27.

16. Hristov M, Erl W, Weber PC. Endothelial progenitor cells: mobilization, differentiation, and homing. Arterioscler Thromb Vasc Biol 2003;23:1185-9.

17. Timmermans F, Plum J, Yöder MC, et al. Endothelial progenitor cells: identity defined? J Cell Mol Med 2009;13:87-102.

18. Yoder MC. Human endothelial progenitor cells. Cold Spring Harb Perspect Med 2012;2:a006692.

19. Blanpain C, Horsley V, Fuchs E. Epithelial stem cells: turning over new leaves. Cell 2007;128:445-58.

20. Rawlins EL. Lung epithelial progenitor cells: lessons from development. Proc Am Thorac Soc 2008;5:675-81.

21. Rock JR, Hogan BL. Epithelial progenitor cells in lung development, maintenance, repair, and disease. Annu Rev Cell Dev Biol 2011;27:493-512.

22. Adamson IY, Bowden DH. Derivation of type 1 epithelium from type 2 cells in the developing rat lung. Lab Invest 1975;32:736-45.

23. Evans MJ, Cabral LJ, Stephens RJ, et al. Transformation of alveolar type 2 cells to type 1 cells following exposure to NO2. Exp Mol Pathol 1975;22:142-50.

24. Dominici M, Le Blanc K, Mueller I, et al. Minimal criteria for defining multipotent mesenchymal stromal cells. The International Society for Cellular Therapy position statement. Cytotherapy 2006;8:315-7.

25. Bianco P, Cao X, Frenette PS, et al. The meaning, the sense and the significance: translating the science of mesenchymal stem cells into medicine. Nat Med 2013;19:35-42.

26. Pourrajab F, Forouzannia SK, Tabatabaee SA. Molecular characteristics of bone marrow mesenchymal stem cells, source of regenerative medicine. Int J Cardiol 2013;163:125-31.

27. Salmikangas $P$, Menezes-Ferreira M, Reischl I, et al. Manufacturing, characterization and control of cellbased medicinal products: challenging paradigms toward commercial use. Regen Med 2015;10:65-78.

28. Deskins DL, Bastakoty D, Saraswati S, et al. Human 
mesenchymal stromal cells: identifying assays to predict potency for therapeutic selection. Stem Cells Transl Med 2013;2:151-8.

29. Matthay MA. Extracellular vesicle transfer from mesenchymal stromal cells modulates macrophage function in acute lung injury: basic science and clinical implications. Am J Respir Crit Care Med 2017;196:1234-6.

30. Rippon HJ, Ali NN, Polak JM, et al. Initial observations on the effect of medium composition on the differentiation of murine embryonic stem cells to alveolar type II cells. Cloning Stem Cells 2004;6:49-56.

31. Rippon HJ, Polak JM, Qin M, et al. Derivation of distal lung epithelial progenitors from murine embryonic stem cells using a novel three-step differentiation protocol. Stem Cells 2006;24:1389-98.

32. Ghaedi M, Calle EA, Mendez JJ, et al. Human iPS cellderived alveolar epithelium repopulates lung extracellular matrix. J Clin Invest 2013;123:4950-62.

33. Wang C, Hei F, Ju Z, et al. Differentiation of urinederived human induced pluripotent stem cells to alveolar type II epithelial cells. Cell Reprogram 2016;18:30-6.

34. Ghaedi M, Mendez JJ, Bove PF, et al. Alveolar epithelial differentiation of human induced pluripotent stem cells in a rotating bioreactor. Biomaterials 2014;35:699-710.

35. Curley GF, Hayes M, Ansari B, et al. Mesenchymal stem cells enhance recovery and repair following ventilatorinduced lung injury in the rat. Thorax 2012;67:496-501.

36. Chimenti L, Luque T, Bonsignore MR, et al. Pretreatment with mesenchymal stem cells reduces ventilatorinduced lung injury. Eur Respir J 2012;40:939-48.

37. Curley GF, Ansari B, Hayes M, et al. Effects of intratracheal mesenchymal stromal cell therapy during recovery and resolution after ventilator-induced lung injury. Anesthesiology 2013;118:924-32.

38. Sepúlveda JC, Tomé M, Fernández ME, et al. Cell senescence abrogates the therapeutic potential of human mesenchymal stem cells in the lethal endotoxemia model. Stem Cells 2014;32:1865-77.

39. Németh K, Leelahavanichkul A, Yuen PS, et al. Bone marrow stromal cells attenuate sepsis via prostaglandin $\mathrm{E}(2)$-dependent reprogramming of host macrophages to increase their interleukin-10 production. Nat Med 2009; 15:42-9.

40. Monsel A, Zhu Y, Gennai S, et al. Therapeutic Effects of Human Mesenchymal Stem Cell-derived Microvesicles in Severe Pneumonia in Mice. Am J Respir Crit Care Med 2015;192:324-36.

41. Gupta N, Krasnodembskaya A, Kapetanaki M, et al.
Mesenchymal stem cells enhance survival and bacterial clearance in murine Escherichia coli pneumonia. Thorax 2012;67:533-9.

42. Gupta N, Su X, Popov B, et al. Intrapulmonary delivery of bone marrow-derived mesenchymal stem cells improves survival and attenuates endotoxin-induced acute lung injury in mice. J Immunol 2007;179:1855-63.

43. Gonzalez-Rey E, Anderson P, González MA, et al. Human adult stem cells derived from adipose tissue protect against experimental colitis and sepsis. Gut 2009;58:929-39.

44. Mei SH, Haitsma JJ, Dos Santos CC, et al. Mesenchymal stem cells reduce inflammation while enhancing bacterial clearance and improving survival in sepsis. Am J Respir Crit Care Med 2010;182:1047-57.

45. Mao M, Wang SN, Lv XJ, et al. Intravenous delivery of bone marrow-derived endothelial progenitor cells improves survival and attenuates lipopolysaccharideinduced lung injury in rats. Shock 2010;34:196-204.

46. Cao JP, He XY, Xu HT, et al. Autologous transplantation of peripheral blood-derived circulating endothelial progenitor cells attenuates endotoxin-induced acute lung injury in rabbits by direct endothelial repair and indirect immunomodulation. Anesthesiology 2012;116:1278-87.

47. Li H, Qiang Y, Wang L, et al. Repair of lipopolysaccharide-induced acute lung injury in mice by endothelial progenitor cells, alone and in combination with simvastatin. Chest 2013;144:876-86.

48. Lam CF, Liu YC, Hsu JK, et al. Autologous transplantation of endothelial progenitor cells attenuates acute lung injury in rabbits. Anesthesiology 2008;108:392-401.

49. Guillamat-Prats R, Puig F, Herrero R, et al. Effect of alveolar type II cells in acute lung injury model. Eur Respir J 2015;46:PA3029.

50. Toya SP, Li F, Bonini MG, et al. Interaction of a specific population of human embryonic stem cellderived progenitor cells with CD11b+ cells ameliorates sepsis-induced lung inflammatory injury. Am J Pathol 2011;178:313-24.

51. Luo CJ, Zhang FJ, Zhang L, et al. Mesenchymal stem cells ameliorate sepsis-associated acute kidney injury in mice. Shock 2014;41:123-9.

52. Choi H, Lee RH, Bazhanov N, et al. Anti-inflammatory protein TSG-6 secreted by activated MSCs attenuates zymosan-induced mouse peritonitis by decreasing TLR2/NF- $\kappa \mathrm{B}$ signaling in resident macrophages. Blood 2011;118:330-8.

53. Krasnodembskaya A, Samarani G, Song Y, et al. Human mesenchymal stem cells reduce mortality and bacteremia 
in gram-negative sepsis in mice in part by enhancing the phagocytic activity of blood monocytes. Am J Physiol Lung Cell Mol Physiol 2012;302:L1003-13.

54. Hall SR, Tsoyi K, Ith B, et al. Mesenchymal stromal cells improve survival during sepsis in the absence of heme oxygenase-1: the importance of neutrophils. Stem Cells 2013;31:397-407.

55. Wang Y, Tan L, Jin J, et al. Non-cultured dermal-derived mesenchymal cells attenuate sepsis induced by cecal ligation and puncture in mice. Sci Rep 2015;5:16973.

56. Raffaghello L, Bianchi G, Bertolotto M, et al. Human mesenchymal stem cells inhibit neutrophil apoptosis: a model for neutrophil preservation in the bone marrow niche. Stem Cells 2008;26:151-62.

57. Zhao Y, Yang C, Wang H, et al. Therapeutic effects of bone marrow-derived mesenchymal stem cells on pulmonary impact injury complicated with endotoxemia in rats. Int Immunopharmacol 2013;15:246-53.

58. Liang Z, Sun J, Wang P, et al. Bone marrow-derived mesenchymal stem cells protect rats from endotoxininduced acute lung injury. Chin Med J (Engl) 2011;124:2715-22.

59. Cóndor JM, Rodrigues CE, Sousa Moreira Rd, et al. Treatment with human Wharton's Jelly-derived mesenchymal stem cells attenuates sepsis-induced kidney injury, liver injury, and endothelial dysfunction. Stem Cells Transl Med 2016;5:1048-57.

60. Chen HH, Lin KC, Wallace CG, et al. Additional benefit of combined therapy with melatonin and apoptotic adipose-derived mesenchymal stem cell against sepsis-

Cite this article as: Guillamat-Prats R, Camprubí-Rimblas M, Bringué J, Tantinyà N, Artigas A. Cell therapy for the treatment of sepsis and acute respiratory distress syndrome. Ann Transl Med 2017;5(22):446. doi: 10.21037/atm.2017.08.28 induced kidney injury. J Pineal Res 2014;57:16-32.

61. Alcayaga-Miranda F, Cuenca J, Martin A, et al. Combination therapy of menstrual derived mesenchymal stem cells and antibiotics ameliorates survival in sepsis. Stem Cell Res Ther 2015;6:199.

62. Kocyildirim E, Cárdenes N, Ting A, et al. The use of GMP-produced bone marrow-derived stem cells in combination with extracorporeal membrane oxygenation in ARDS: an animal model. ASAIO J 2017;63:324-32.

63. Güldner A, Maron-Gutierrez T, Abreu SC, et al. Expanded endothelial progenitor cells mitigate lung injury in septic mice. Stem Cell Res Ther 2015;6:230.

64. Xu X, Yang J, Li N, et al. Role of endothelial progenitor cell transplantation in rats with sepsis. Transplant Proc 2015;47:2991-3001.

65. Fan H, Goodwin AJ, Chang E, et al. Endothelial progenitor cells and a stromal cell-derived factor- $1 \alpha$ analogue synergistically improve survival in sepsis. Am J Respir Crit Care Med 2014;189:1509-19.

66. Zheng G, Huang L, Tong H, et al. Treatment of acute respiratory distress syndrome with allogeneic adiposederived mesenchymal stem cells: a randomized, placebocontrolled pilot study. Respir Res 2014;15:39.

67. Galstyan GM, Makarova PM, Parovichnikova EN. Use of mesenchymal stromal stem cells for the treatment of sepsis. Anesteziol Reanimatol 2015;60:59-65.

68. Wilson JG, Liu KD, Zhuo H, et al. Mesenchymal stem (stromal) cells for treatment of ARDS: a phase 1 clinical trial. Lancet Respir Med 2015;3:24-32. 